\title{
Performance Related Hemodynamic Responses to an Aerobic Exercise Across Different Stages of the Menstrual Cycle
}

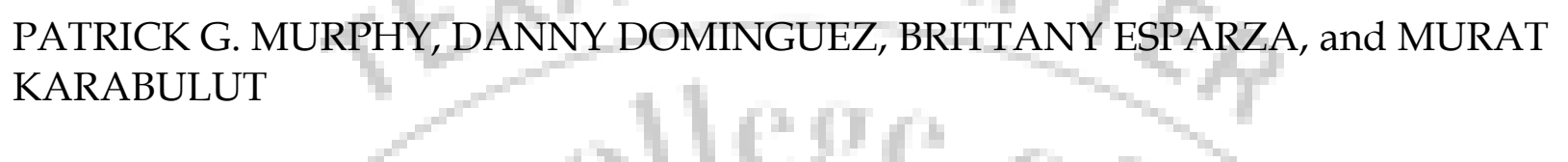

Health and Human Performance Exercise Science Laboratory; Department of Health and Human Performance; University of Texas - Rio Grande Valley; Brownsville, TX

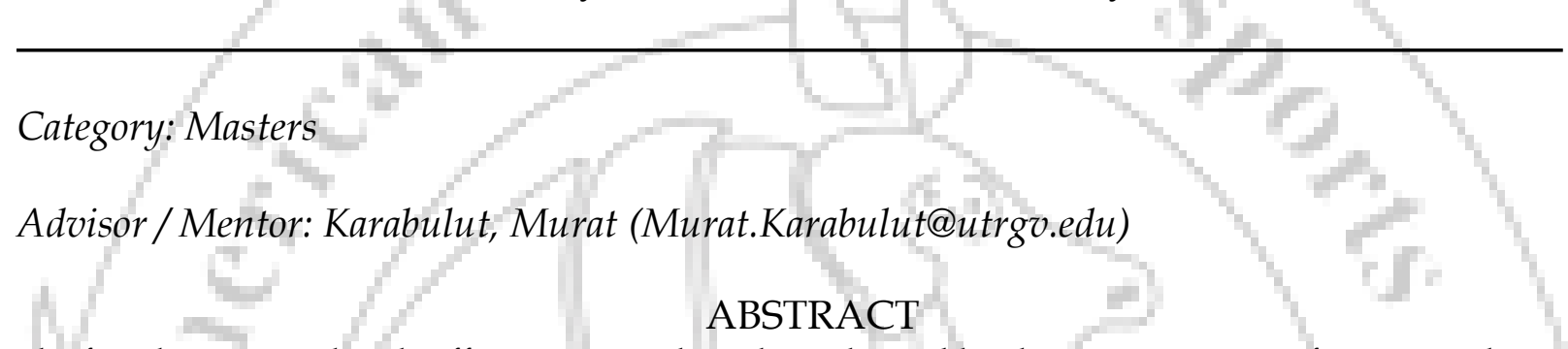

The female menstrual cycle affects various physiological variables that may impact performance. The cyclical nature could lead to differences across the entire cycle and therefore it may be beneficial to recommend the timing of an individuals cycle in coherence with a performance event. PURPOSE: To examine the physiological changes, especially body water and hemodynamic measures that occur in females across their respective menstrual cycles in response to aerobic performance. METHODS: Eleven females (age $=21.2 \pm 1.4 \mathrm{yrs}$ ) performed four sessions of aerobic activity at $75 \%$ of their calculated VO2 max as determined by the Bruce Protocol. The speed for each subject's aerobic activity was then calculated using the ACSM metabolic running equation. The four sessions of aerobic activity were identical in format and performed on days 1, 7, 14, and 21 of each subject's menstrual cycle. Sessions began with measurements of body weight, body fat, heart rate (HR), and blood pressure (BP). Extracellular fluid (ECF), intracellular fluid (ICF), and fat-free mass (FFM was also recorded via single-frequency and multiple-frequency bio-electrical impedance analysis (BIA) prior to aerobic activity. Subjects then completed 30 minutes of aerobic exercise at the calculated speed to yield an intensity of $75 \% \mathrm{VO} 2$ max. Following the aerobic event, all measures taken pre-exercise, as well as RPE, were measured again at three separate time points post-exercise (post-0 min, post-15min, and post-30min) while HR was monitored continuously. RESULTS: Day one systolic BP significantly correlated with day one $\triangle E C F$ at multiple time points $(r=-0.619$ to $-0.680, p<0.05)$. HR also had significant correlations on day one with $\triangle E C F$ and $\triangle I C F(r$ $=-0.610, p<0.05 ; \mathrm{r}=0.609, \mathrm{p}<0.05$ ). Day 7 had significant correlations between BP (systolic and diastolic) and ICF $(r=-0.610$ to $-0.672, \mathrm{p}<0.05)$ at multiple time points. CONCLUSIONS: The results showed that hemodynamic variables had notable correlations throughout most performance-related measures and across the different days of the menstrual cycle. From Day 1 to 7 there is an observable shift in correlation from BP and ECF to BP and ICF. This apparent shift in fluid may be due to the physiological responses associated with progressing through one's cycle from the menstrual to luteal phase in which a significant amount of body water is lost. These results demonstrate that there may be different responses to aerobic performance throughout the different phases of the menstrual cycle, but further investigation is required to make any kind of recommendations. 